\title{
Geodésicas e Momento Angular Constante no espaço Anti-de Sitter $(2,3)$
}

\author{
Samuel A. Wainer ${ }^{1}$ \\ Instituto Tecnológico de Aeronáutica, São José dos Campos, SP
}

Resumo. Considere $M^{a d S L}$ o Espaço de Anti-de Sitter Lorentziano de assinatura $(2,3)$. Neste trabalho demontraremos o fato de que se o movimento de uma partícula restrita à $M^{a d S L}$, sem ação de nenhuma força detetável por observadores em $M^{a d S L}$, acontece sob momento angular constante, visto em $\mathbb{R}^{2,3}$, então essa trajetória acontece em uma geodésica tipo-tempo. Também demonstraremos que qualquer trajetória em um geodésica na estrutura $M^{\text {adSL }}$ implica em movimento sob momento angular constante, visto em $\mathbb{R}^{2,3}$.

Palavras-chave. Anti-de Sitter, Momento Angular, Geodésicas, Relatividade Geral.

\section{Introdução}

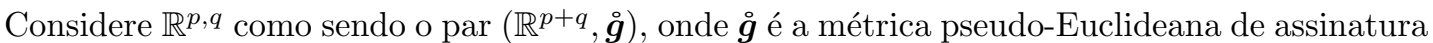
$(p, q)$. No que segue, denotaremos por $S O(p, q)$ o grupo pseudo-ortogonal em $\mathbb{R}^{p, q}$.

O espaço de Anti-de Sitter $M=S O(2,3) / S(1,3)$ de assinatura $(2,3)$ é uma brana, ou seja uma subvariedade, na estrutura $\mathbb{R}^{2,3}$. Definimos o espaço de Anti-de Sitter Lorentziano de assinatura $(2,3)$ como a estrutura $M^{a d S L}=\left(M, \boldsymbol{g}, D, \tau_{\boldsymbol{g}}, \uparrow\right)$, onde se $i: M \rightarrow \mathbb{R}^{5}$ é a aplicação inclusão, então

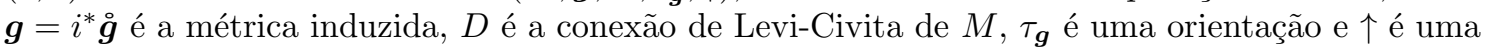
orientação no tempo em $M$ (para mais detalhes, consulte $[8,9]$ ).

Além disso, $(M, \boldsymbol{g})$ é uma pseudo-esfera na estrutura $\mathbb{R}^{2,3}$ e é um espaço-tempo com curvatura Riemanniana constante. O grupo $S O(2,3)$ age transitivamente em $S O(2,3) / S O(1,3)$, que é, portanto, um espaço homogêneo (para $S O(2,3)$ ).

O espaço de Anti-de Sitter e também o espaço de de Sitter vem sendo usados por muitos físicos $[4,5]$ como espaço alternativo para movimento de partículas e campos ao invés do espaçotempo de Minkowski $\mathcal{M}$. Como é bem conhecido, o movimento natural de uma partícula livre de massa $m$ em $\mathcal{M}$ acontece sob momento linear constante $\boldsymbol{p}=m \gamma_{*}=$ cte, onde $\gamma: \mathbb{R} \rightarrow M$ é uma curva tipo-tempo. A questão que surge aqui é a seguinte:

Qual é o movimento natural de uma partícula livre na estrutura $(M, \boldsymbol{g})$ ?

Uma sugestão natural para o espaço de de Sitter [2] é que tal movimento acontece sob momento angular constante $\boldsymbol{L}$ determinado por observadores do espaço ambiente em que $M$ está mergulhado. Guiados por tal ideia, apresentamos aqui o seguinte resultado:

a) Se uma partícula viaja em uma geodésica na estrutura $M^{a s S L}$, então seu momento angular $\boldsymbol{L}$ em $\mathbb{R}^{2,3}$ é constante.

\footnotetext{
${ }^{1}$ wainer@ita.br.
} 
b) Se uma partícula de massa $m$ restrita a se mover em $M$ com momento angular constante em $\mathbb{R}^{2,3}$, então seu movimento quando visto por um observador na brana $M$ será descrito por uma geodésica tipo-tempo na estrutura $M^{a d S L}$.

Este trabalho está estruturado da seguinte maneira: na Seção 2 fixamos as notações necessárias, descrevemos o espaço Anti-de Sitter $M$ como uma pseudo-esfera mergulhada em $\mathbb{R}^{2,3}$ e apresentamos a equação de movimento geodésico (de uma partícula livre de massa $m$ ) na estrutura $M^{a d S L}$. Na Seção 3 apresentamos a equação de movimento que uma partícula de massa $m$ restrita a se mover em $M$ com momento angular constante em $\mathbb{R}^{2,3}$ e com isso enunciamos o resultado de que os conceitos de momento angular constante e movimento geodésico são equivalentes na estrutura $M^{a d S L}$. Por fim, na Seção 4 apresentamos nossas conclusões e perspectivas de trabalhos futuros.

\section{Geodésicas no Espaço Anti-de Sitter $(2,3)$}

Primeiramente vamos apresentar a descrição do espaço Anti-de Sitter de assinatura $(2,3), M$, como uma pseudo-esfera de raio $l$ dentro da estrutura $\mathbb{R}^{2,3}$.

Seja $\left(X^{-1}, X^{0}, X^{1}, X^{2}, X^{3}\right)$ coordenadas cartesianas globais de $\mathbb{R}^{2,3}$. A equação representando a pseudo-esfera é

$$
\left(X^{-1}\right)^{2}+\left(X^{0}\right)^{2}-\left(X^{1}\right)^{2}-\left(X^{2}\right)^{2}-\left(X^{3}\right)^{2}=l^{2} .
$$

Aplicando a projeção estereográfica em $M$ podemos introduzir coordenadas conformes $\left\{x^{\mu}\right\} \mathrm{em}$ M. Imediatamente temos que ${ }^{2}$ :

onde

$$
\boldsymbol{g}=i^{*} \stackrel{\circ}{\boldsymbol{g}}=g_{\mu \nu} d x^{\mu} \otimes d x^{\nu}=\Omega^{2} \eta_{\mu \nu} d x^{\mu} \otimes d x^{\nu},
$$

$$
\begin{gathered}
g_{\mu \nu}=\Omega^{2} \eta_{\mu \nu}, \quad \sigma^{2}=\eta_{\mu \nu} x^{\mu} x^{\nu}, \\
\Omega=\frac{4 l^{2}}{4 l^{2}+\sigma^{2}}=\frac{1}{1+\frac{\sigma^{2}}{4 l^{2}}}, \quad \mathrm{e} \\
X^{\mu}=\Omega x^{\mu}, \quad X^{-1}=l \Omega\left[\frac{\sigma^{2}}{4 l^{2}}-1\right]=l(1-2 \Omega) .
\end{gathered}
$$

Agora, escrevendo $D_{\partial_{\mu}} \partial_{\nu}=\Gamma_{\mu \nu}^{\alpha} \partial_{\alpha}$, os coeficientes de conexão não nulos são:

$$
\begin{array}{llll}
\Gamma_{00}^{0}=-\frac{\Omega}{2 l^{2}} x^{0}, & \Gamma_{01}^{0}=\frac{\Omega}{2 l^{2}} x^{1}, & \Gamma_{02}^{0}=\frac{\Omega}{2 l^{2}} x^{2}, & \Gamma_{03}^{0}=\frac{\Omega}{2 l^{2}} x^{3}, \\
\Gamma_{11}^{0}=-\frac{\Omega}{2 l^{2}} x^{0}, & \Gamma_{22}^{0}=-\frac{\Omega}{2 l^{2}} x^{0}, & \Gamma_{33}^{0}=-\frac{\Omega}{2 l^{2}} x^{0}, & \Gamma_{00}^{1}=\frac{\Omega}{2 l^{2}} x^{1}, \\
\Gamma_{01}^{1}=-\frac{\Omega}{2 l^{2}} x^{0}, & \Gamma_{11}^{1}=\frac{\Omega}{2 l^{2}} x^{1}, & \Gamma_{12}^{1}=\frac{\Omega}{2 l^{2}} x^{2}, & \Gamma_{13}^{1}=\frac{\Omega}{2 l^{2}} x^{3}, \\
\Gamma_{22}^{1}=-\frac{\Omega}{2 l^{2}} x^{1}, & \Gamma_{33}^{1}=-\frac{\Omega}{2 l^{2}} x^{1}, & \Gamma_{00}^{2}=\frac{\Omega}{2 l^{2}} x^{2}, & \Gamma_{02}^{2}=-\frac{\Omega}{2 l^{2}} x^{0}, \\
\Gamma_{11}^{2}=-\frac{\Omega}{2 l^{2}} x^{2}, & \Gamma_{12}^{2}=\frac{\Omega}{2 l^{2}} x^{1}, & \Gamma_{22}^{2}=\frac{\Omega}{2 l^{2}} x^{2}, & \Gamma_{23}^{2}=\frac{\Omega}{2 l^{2}} x^{3}, \\
\Gamma_{33}^{2}=-\frac{\Omega}{2 l^{2}} x^{2}, & \Gamma_{00}^{3}=\frac{\Omega}{2 l^{2}} x^{3}, & \Gamma_{03}^{3}=-\frac{\Omega}{2 l^{2}} x^{0}, & \Gamma_{11}^{3}=-\frac{\Omega}{2 l^{2}} x^{3}, \\
\Gamma_{13}^{3}=\frac{\Omega}{2 l^{2}} x^{1}, & \Gamma_{22}^{3}=-\frac{\Omega}{2 l^{2}} x^{3}, & \Gamma_{23}^{3}=\frac{\Omega}{2 l^{2}} x^{2}, & \Gamma_{33}^{3}=\frac{\Omega}{2 l^{2}} x^{3} .
\end{array}
$$

\footnotetext{
${ }^{2} \mathrm{~A}$ matriz com entradas $\eta_{\mu \nu}$ é uma matriz diagonal $\operatorname{diag}(1,-1,-1,-1)$.
} 
Seja $\gamma: I \rightarrow M, s \mapsto \gamma(s)$ uma geodésica tipo-tempo em $M$. Nós sabemos que o campo vetorial tangente $\gamma_{*}(s)=\left.\frac{d \boldsymbol{x}^{\alpha} \circ \gamma(s)}{d s} \frac{\partial}{\partial x^{\alpha}}\right|_{\gamma}$ satisfaz $D_{\gamma_{*}} \gamma_{*}=0$, que escrito em coordenadas é

$$
\frac{d^{2} x^{\alpha}}{d s^{2}}+\Gamma_{\mu \nu}^{\alpha} \frac{d x^{\mu}}{d s} \frac{d x^{\nu}}{d s}=0
$$

Usando os coeficientes de conexão dados pela Eq.(1) obtemos a equação da geodésica para o espaço de Anti-de Sitter de assinatura $(2,3)$ :

$$
\frac{d^{2} x^{\alpha}}{d s^{2}}-\frac{\Omega}{l^{2}} x_{\mu} \frac{d x^{\mu}}{d s} \frac{d x^{\alpha}}{d s}+\frac{\Omega}{2 l^{2}} x^{\alpha} \frac{d x_{\mu}}{d s} \frac{d x^{\mu}}{d s}=0 .
$$

\section{Momento Angular Constante e Geodésicas}

Seja

$$
\left\{\boldsymbol{E}_{A}:=\frac{\partial}{\partial X^{A}}\right\}, A=-1,0,1,2,3
$$

a base canônica de $T \mathbb{R}^{2,3}$ e seja $\left\{E^{A}:=d X^{A}\right\}$ uma base de $T^{*} \mathbb{R}^{2,3}$ dual a $\left\{\boldsymbol{E}_{A}\right\}$.

A métrica $\stackrel{g}{g}$ em $\mathbb{R}^{2,3}$ é dada por

$$
\stackrel{\circ}{\boldsymbol{g}}=\boldsymbol{\eta}_{A B} E^{A} \otimes E^{B},
$$

onde é a matriz com entradas $\boldsymbol{\eta}_{A B}$ é a matriz diagonal $\operatorname{diag}(1,1,-1,-1,-1)$.

Além disso seja

$$
\stackrel{\circ}{g}=\eta^{A B}=\boldsymbol{E}_{A} \otimes \boldsymbol{E}_{B}
$$

a métrica do fibrado cotangente (com $\left.\eta^{A B} \boldsymbol{\eta}_{B C}=\delta_{C}^{A}\right)$. Finalmente seja $\left\{E_{A}\right\}$ a base recíproca de $\left\{E^{A}\right\}$, i.e., $\stackrel{g}{g}\left(E^{A}, E_{B}\right)=\delta_{B}^{A}$. Introduzimos a base $\left\{\mathcal{E}_{A}\right\}$ de $\mathbb{R}^{5}$ e consideramos a identificação usual $E_{A}(p) \simeq E_{A}\left(p^{\prime}\right) \simeq \mathcal{E}_{A}, \forall p, p^{\prime} \in \mathbb{R}^{5}$

Sejam $\boldsymbol{X}=X^{A} \mathcal{E}_{A}$ o covetor posição, $\boldsymbol{P}=m \ddot{X}^{B} \mathcal{E}_{B}$ o covetor momento linear em $\mathbb{R}^{2,3} \mathrm{e}$ $\boldsymbol{L}=\boldsymbol{X} \wedge \boldsymbol{P}$ o momento angular de uma partícula de massa $m \mathrm{em} \mathbb{R}^{2,3}$. Se a partícula está se movendo livremente ${ }^{3}$ na subvariedade $M$, uma hipótese natural é que o momento angular em $\mathbb{R}^{2,3}$ desta partícula seja uma constante de movimento.

$\boldsymbol{L}=\boldsymbol{c t e}$ implica imediatamente em

$$
\frac{1}{2}\left(X^{A} \ddot{X}^{B}-\ddot{X}^{A} X^{B}\right) \mathcal{E}_{A} \wedge \mathcal{E}_{B}=0 .
$$

Assim, para $\alpha, \beta=0,1,2,3$ teremos $X^{\alpha} \ddot{X}^{\beta}-\ddot{X}^{\alpha} X^{\beta}=0$, i.e.,

$$
x^{\alpha}\left(-\frac{\Omega}{l^{2}} x_{\mu} \frac{d x^{\mu}}{d s} \frac{d x^{\beta}}{d s}+\frac{d^{2} x^{\beta}}{d s^{2}}\right)-\left(-\frac{\Omega}{l^{2}} x_{\mu} \frac{d x^{\mu}}{d s} \frac{d x^{\alpha}}{d s}+\frac{d^{2} x^{\alpha}}{d s^{2}}\right) x^{\beta}=0 .
$$

Também, da equação $X^{\alpha} \ddot{X}^{-1}-\ddot{X}^{\alpha} X^{-1}=0$, segue que

$$
\begin{gathered}
-(2 \Omega-1)\left(\frac{d^{2} x^{\alpha}}{d s^{2}}+\frac{1}{l^{2}} \Omega x_{i} \frac{d x^{i}}{d s} \frac{d x^{\alpha}}{d s}\right) \\
+\frac{1}{2 l^{2}} \Omega\left(\frac{1}{l^{2}} \Omega x_{i} x_{j} \frac{d x^{i}}{d s} \frac{d x^{j}}{d s}+\frac{d x_{i}}{d s} \frac{d x^{i}}{d s}+x_{i} \frac{d^{2} x^{i}}{d s^{2}}\right) x^{\alpha}=0 .
\end{gathered}
$$

\footnotetext{
${ }^{3}$ Do ponto de vista físico, dizer que se move livremente quer dizer que observadores morando em $M$ não podem detectar nenhuma força agindo na partícula.
} 
Estas são as equações do movimento de acordo com a estrutura $M^{a d S L}$. Fixadas tais notações e hipóteses, podemos demonstrar a seguinte proposição:

\section{Proposição 3.1.}

a) Se uma partícula viaja em uma geodésica na estrutura $M^{a s S L}$, então seu momento angular $\boldsymbol{L}$ em $\mathbb{R}^{2,3}$ é constante.

b) Se uma partícula de massa $m$ restrita a se mover em $M$ com momento angular constante em $\mathbb{R}^{2,3}$, então seu movimento quando visto por um observador na brana $M$ será descrito por uma geodésica tipo-tempo na estrutura $M^{a d S L}$.

Tal resultado segue da comparação entre as Eqs. (5), (6), com a Eq. (3).

\section{Conclusões}

O espaço de Anti-de Sitter e também o espaço de de Sitter vem sendo estudados como um espaço natural para o movimento de partículas e campos ao invés do espaço-tempo de Minkowski $\mathcal{M}$. Discutimos essas propriedades para o espaço de de Sitter em [7,8]. Recentemente [6], demonstramos que usando o formalismo do fibrado de Clifford [9], a hipótese que uma partícula movendo livremente no espaço de de Sitter com momento angular constante visto do espaço ambiente leva naturalmente à uma equação de Dirac [1] nesse espaço. Com as afirmações demonstradas na Proposição 3.1, sobre a equivalência entre geodésicas e momento angular constante visto do espaço ambiente, podemos esperar que o mesmo é válido para o Espaço Anti-de Sitter, isto é, utilizando o formalismo do fibrado de Clifford, podemos obter uma equação de Dirac, o que pretendemos demonstrar em trabalhos futuros.

\section{Referências}

[1] Dirac, P. A. M., The Electron Wave Equation in De-Sitter Space, Ann. Math. 36, 657-669, 1935.

[2] Gürsey, F., Introduction to Group Theory, in DeWitt, C. and DeWitt, B. (eds.), Relativity, Groups and Topology, pp 91-161, Gordon and Breach, New York, 1964.

[3] Hawking, S. W. e Ellis, G. F. R., The Large Scale Structure of Space-Time, Cambridge Univ. Press, Cambridge, 1973.

[4] Pereira, J. G. e Sampson, A. C., de Sitter Geodesics: Reappraising the Notion of Motion, Gen Relativ Gravit, 44, 1299-1308, (2012).

[5] Pereira, J. G., Sampson, A. C. e Savi L. L., de Sitter Transitivity, Con-formal Transformations and Conservation Laws, Int. J. Mod. Phys, D 23, 1450035 (2014).

[6] Rodrigues, W. A. Jr., Wainer, S. A., Rivera-Tapia, M., Notte-Cuello, E. A., Kondrashuk, I., A Clifford Bundle Approach to the Wave Equation of a Spin 1/2 Fermion in the de Sitter Manifold, Adv. Appl. Clifford Algebras, 253-277 (2016).

[7] Rodrigues, W. A. Jr. and Wainer, S. A., Notes on Conservation Laws, Equations of Motion and Particle Field in Lorentzian and Teleparallel de Sitter Spacetime Structures, Adv. Math. Phys. 2016, 5465263 (2016) 
[8] Rodrigues, W. A. Jr., Wainer, S.A. On the Motion of a Free Particle in the de Sitter Manifold. Adv. Appl. Clifford Algebras 27, 1761-1767 (2017).

[9] Rodrigues, W. A. Jr. and Capelas de Oliveira, E., The Many Faces of Maxwell, Dirac and Einstein Equation,. A Clifford Bundle Approach (second edition, revised and enlarged), Lecture Notes in Physics 922, Springer, Heidelberg, 2016. 